\title{
Measuring charge trap occupation and energy level in CdSe/ZnS quantum dots using a scanning tunneling microscope
}

\section{Citation}

Hummon, M. R., A. J. Stollenwerk, V. Narayanamurti, P. O. Anikeeva, M. J. Panzer, V. Wood, and V. Bulović. 2010. "Measuring Charge Trap Occupation and Energy Level in CdSe/ZnS Quantum Dots Using a Scanning Tunneling Microscope." Physical Review B 81 (11). https://doi.org/10.1103/ physrevb.81.115439.

\section{Permanent link}

http://nrs.harvard.edu/urn-3:HUL.InstRepos:41412215

\section{Terms of Use}

This article was downloaded from Harvard University's DASH repository, and is made available under the terms and conditions applicable to Other Posted Material, as set forth at http:// nrs.harvard.edu/urn-3:HUL.InstRepos:dash.current.terms-of-use\#LAA

\section{Share Your Story}

The Harvard community has made this article openly available.

Please share how this access benefits you. Submit a story.

\section{Accessibility}




\title{
Measuring charge trap occupation and energy level in CdSe/ZnS quantum dots using a scanning tunneling microscope
}

\author{
M. R. Hummon, * A. J. Stollenwerk, and V. Narayanamurti \\ School of Engineering and Applied Sciences, Harvard University, Cambridge, Massachusetts 02138, USA \\ P. O. Anikeeva, M. J. Panzer, V. Wood, and V. Bulović \\ Laboratory of Organic Optics and Electronics, Massachusetts Institute of Technology, Cambridge, Massachusetts 02139, USA \\ (Received 22 July 2009; revised manuscript received 17 February 2010; published 22 March 2010)
}

\begin{abstract}
We use a scanning tunneling microscope to probe single-electron charging phenomena in individual CdSe/ $\mathrm{ZnS}$ (core/shell) quantum dots (QDs) at room temperature. The QDs are deposited on top of a bare Au thin film and form a double-barrier tunnel junction (DBTJ) between the tip, QD, and substrate. Analysis of roomtemperature hysteresis in the current-voltage (IV) tunneling spectra, is consistent with trapped charge(s) presenting an additional potential barrier to tunneling, a measure of the Coulomb blockade. The paper describes the first direct electrical measurement of the trap-state energy on individual QDs. Manipulation of the charge occupation of the QD, verified by measuring the charging energy, $(61.4 \pm 2.4) \mathrm{meV}$, and analysis of the DBTJ, show trap states $\sim 1.09 \mathrm{eV}$ below the QD conduction-band edge. In addition, the detrapping time, a measure of the tunneling barrier thickness, is determined to have an upper time limit of $250 \mathrm{~ms}$. We hypothesize that the charge is trapped in a quantum-dot surface state.
\end{abstract}

DOI: 10.1103/PhysRevB.81.115439

PACS number(s): 73.21.La, 73.20.At, 72.20.Jv

\section{INTRODUCTION}

To extend the understanding of charge injection in devices containing quantum-dot (QD) monolayers, we use a scanning tunneling microscope (STM) to inject charge into individual QDs that form close-packed monolayer islands on a conducting surface. Our observations include quantum-dot charge-trapping/detrapping events and Coulomb blockade (CB) at room temperature. These measurements yield the first electrical detection of the trap-state energy level. Our analysis of the distribution of applied bias across the doublebarrier tunnel junction (DBTJ) formed between the STM tip, $\mathrm{QD}$, and $\mathrm{Au}$ substrate provides an explanation for the trapping/detrapping dynamics.

The CdSe/ZnS QDs studied in this paper, typical of core/ shell QDs, have been extensively utilized in optoelectronic device demonstrations. ${ }^{1-5}$ Their brilliant emission and near unity internal quantum efficiency ${ }^{6}$ make them attractive components for light-emitting devices. ${ }^{1,3-5,7}$ However, the devices can suffer from a large inefficiency because of an imbalance in charge injection and subsequent quenching. ${ }^{8}$ Earlier studies have probed charge transport in multilayer ${ }^{6,7,9,10}$ and monolayer ${ }^{5,11,12}$ QD films spanning two macroscopic electrodes. STM studies of colloidal quantum dots include shell tunneling and filling spectroscopy, ${ }^{13-18}$ manipulation of the DBTJ structure, ${ }^{19-21}$ and single-electron charging. ${ }^{22-24}$

Averin and Likharev first described how Coulomb blockade arises during single-electron tunneling events. ${ }^{25}$ They deduced that if an electron tunneling through a very small capacitance junction encounters a charge of $-e / 2<Q<e / 2$, then electrons will be blocked from tunneling until that charge has been shunted. This period of zero conductance was termed $\mathrm{CB}$, and in the last two decades has been thoroughly investigated at low temperatures $(T<4 \mathrm{~K})$. Experiments on metal islands show a CB around zero bias, ${ }^{26}$ and as the bias is increased, the characteristic Coulomb staircase emerges due to charges piling up on the island. Experiments on semiconducting QDs show a zero-conductance region equal to $E_{g}+2 E_{c}$, where $E_{g}$ is the band gap of the material and $E_{c}$ is the Coulomb potential of a charge on the QD, also referred to as the charging energy; ${ }^{13}$ at higher biases they also exhibit a Coulomb staircase (CS).

The first CB studies on nanocrystals were done on silicon because of their possible application in QD-based memory. ${ }^{27}$ Most CB studies on nanocrystals show CS in the currentvoltage $(I V)$ spectra, resulting from electrons filling the conduction states of the QD. In this paper, we describe the first observation and analysis of $\mathrm{CB}$ found in the hysteresis of subsequent $I V$ measurements in single $\mathrm{CdSe} / \mathrm{ZnS}$ QDs. The hysteresis is consistent with measuring the $I V$ spectra of a single QD in two different charge states, likely due to a localized trapped charge. Our results also provide an upper time limit of $250 \mathrm{~ms}$ for the charge that remains trapped on the $\mathrm{CdSe} / \mathrm{ZnS} \mathrm{QD}$ at room temperature, when the QD is exposed to the electric field generated by a voltage drop between the STM tip and the Au-film substrate, on which the QD is located, of 1.55 V. An analysis of the voltage division across the DBTJ shows that this bias corresponds to trap states located $\sim 460 \mathrm{meV}$ below the QD core conductionband edge. We hypothesize that the trapped charge is in a surface state and provide a possible mechanism for trapping and detrapping the charge by evaluating the band-energy alignment of the surface states and the Au-film Fermi level.

\section{EXPERIMENTAL METHODS}

\section{A. Scanning tunneling microscope}

Our microscope is home built, and for this study we operated it under ultrahigh vacuum (UHV) $\left(<1 \times 10^{-9}\right.$ Torr), room-temperature conditions. The STM is suspended from 
the UHV chamber by springs, with stationary copper fins that surround opposing magnets dampening any STM head vibration oscillations. The entire UHV system is vibration isolated from the laboratory by pneumatic dampening legs and is situated in a sound and stray-field-reduced room. Coarse approach and $\mathrm{x}-\mathrm{y}$ positioning is achieved with attocube nanopositioners, ANPx51 and ANPz51, and actuated by the attocube ANC-150 waveform generator. XY scanning and fine-height control is achieved with a piezoelectric ceramic tube $(12.7 \mathrm{~mm} \times$ OD $3.1 \mathrm{~mm})$ with a scan range of $3 \mu \mathrm{m}^{2}$ and a height range of $500 \mathrm{~nm}$. We use a home built, $10^{8}$ current preamplifier, located $0.5 \mathrm{~m}$ from the tip. The high voltage for the scanning piezo is provided by a RHKSPM1000 controller and operated with RHK XPMPRO2.0 software.

To form atomically sharp STM tips, we etch $150 \mu \mathrm{m} \mathrm{W}$ wire in a $5 \mathrm{M}$ potassium hydroxide $(\mathrm{KOH})$ solution. The $\mathrm{W}$ wire is etched with a $5 V_{r m s}$ bias while in contact with a $\mathrm{KOH}$ film that is suspended across a Au wire ring. During STM operations of imaging and spectroscopy, tunneling current is measured at the grounded STM tip while bias is applied to the Au-film substrate.

\section{B. Sample preparation}

In this study, we investigate the charging response of $\mathrm{CdSe} / \mathrm{ZnS}$ core/shell quantum dots provided by QD Vision, Inc. The QD synthesis follows previously published work by Dabbousi et al. ${ }^{28}$ The ligands capping the QD are standard aliphatic hydrocarbons, with a mixture of C10-C18 chain lengths. The QDs are crashed twice in methanol and redispersed in chloroform. The peak photoluminescence wavelength is $\lambda \equiv 614 \mathrm{~nm}$ with a full-width half maximum (FWHM) of $32 \mathrm{~nm}$, and a $95 \%$ quantum yield in solution. A submonolayer of QDs is dispersed on a poly(dimethylsiloxane) stamp by spin casting and transferred to a $\mathrm{Au}$ thin film by contact printing, as described by Kim et al. ${ }^{29}$ The Au thin film was formed from template stripped thermal evaporated films, similar to work done by Blackstock et al. on template stripped platinum surfaces. ${ }^{30}$

The samples are annealed at $120^{\circ} \mathrm{C}$ for $1 \mathrm{~h}$ in low vacuum conditions (8 Torr) in a Centurion Qex furnace (DENTSPLY Ceramco) to evaporate some of the capping ligands from the surface of the QDs. ${ }^{6,31}$ Atomic force microscopy (AFM) images, shown in Fig. 2 (obtained on a DI Dimension 3000 in tapping mode) of as-deposited and annealed QD submonolayers showed a reduction in the distance between the top of the QD and the Au surface for the annealed QDs of $0.6 \mathrm{~nm}$, suggesting that some of the ligands left the QDs. STM imaging greatly improved on annealed samples, most likely due to the increased conductivity between the QD layer and the substrate. Photoluminescence studies of annealed QDs show a slight redshift of the peak wavelength due to an increase in wave-function overlap between adjacent QDs; however, broad infrared emission is also detected, indicating an increase in the number of trap states which is likely due to the loss of capping ligands during the anneal. ${ }^{32}$

\section{Tunneling current-sample bias spectroscopy}

Tunneling spectra on bulk semiconductors typically record a zero-conductance region (ZCR) attributed to the
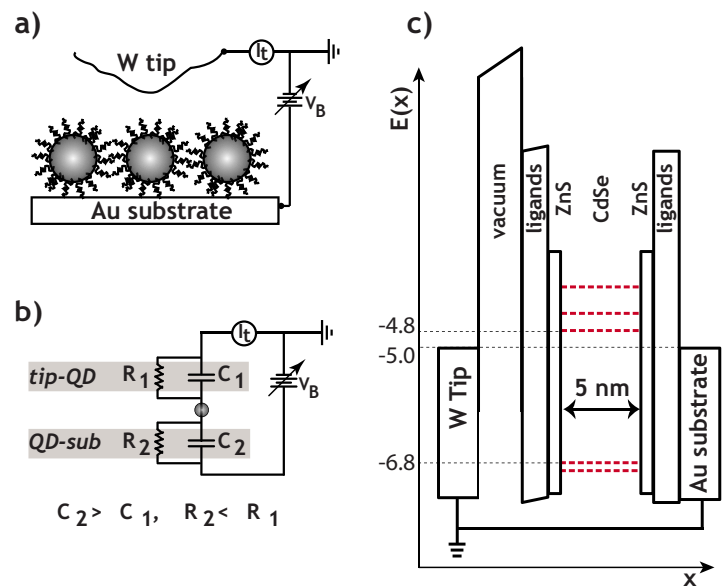

FIG. 1. (Color online) Depictions of (a) STM tip over a QD on a Au-film substrate and (b) equivalent circuit where $C_{1}, R_{1}$ and $C_{2}$, $R_{2}$ are the tunnel barriers between the tip-QD and QD-substrate, respectively. (c) Energy-band diagram of a CdSe/ZnS QD with ligand capping layer on a Au-film substrate with both the substrate and STM tip grounded.

electronic band gap of the material. The ZCR in semiconductor nanostructures, where energy confinement is nonnegligible, spans the electronic band gap and is further increased by the electron-hole Coulomb attraction energy. ${ }^{33}$ Compounding the complexity of measuring the band gap of the QD with tunneling spectroscopy is that the QD is not ohmically contacted by either the tip or substrate. ${ }^{34}$ In fact, the DBTJ formed between the STM tip, the CdSe/ZnS QD, and the $\mathrm{Au}$ surface [energy-band diagram in Fig. 1(b)], causes the bias applied to the substrate $\left(V_{B}\right)$, relative to the tip, to be distributed between the two junctions, inversely proportional to the capacitance of each junction, ${ }^{35}$

$$
\begin{aligned}
& V_{t i p-\mathrm{QD}}=V_{B} \frac{C_{\mathrm{QD}-s u b}}{C_{\mathrm{QD}-s u b}+C_{t i p-\mathrm{QD}}}, \\
& V_{\mathrm{QD}-s u b}=V_{B} \frac{C_{t i p-\mathrm{QD}}}{C_{\mathrm{QD}-s u b}+C_{t i p-\mathrm{QD}}} .
\end{aligned}
$$

An accurate measurement of the single-particle gap for a QD is restricted by the relative capacitance of the QD to the substrate $\left(C_{\mathrm{QD}-\mathrm{sub}}\right)$ and the tip to the QD $\left(C_{\text {tip-QD }}\right) .{ }^{16} \mathrm{We}$ assume that the QD-substrate junction remains constant for a single QD and is fairly uniform for a monolayer of QDs on a surface, and therefore $C_{\mathrm{QD}-s u b}$ is constant for a sample. $C_{\text {tip-QD }}$ is primarily determined by the distance between the STM tip and the QD. This is controlled by the STM feedback loop which maintains a set-point current for a given $V_{B}$. If $C_{\text {tip-QD }} \ll C_{\mathrm{QD}-\text { sub }}$ (the tip is far from the QD), then most of the bias drops between the tip and the QD, and the Fermi level of the substrate $\left(E_{F, s u b}\right)$ remains constant relative to the QD. The STM tip-QD-substrate system thus forms an asymmetric DBTJ.

By moving the tip closer to the QD, a symmetric DBTJ can be established, where $C_{\text {tip-QD }}$ approaches $C_{\mathrm{QD}-s u b}$. Two issues arise in a symmetric DBTJ: (1) unipolar transport may occur, where, at both positive and negative biases, electrons 


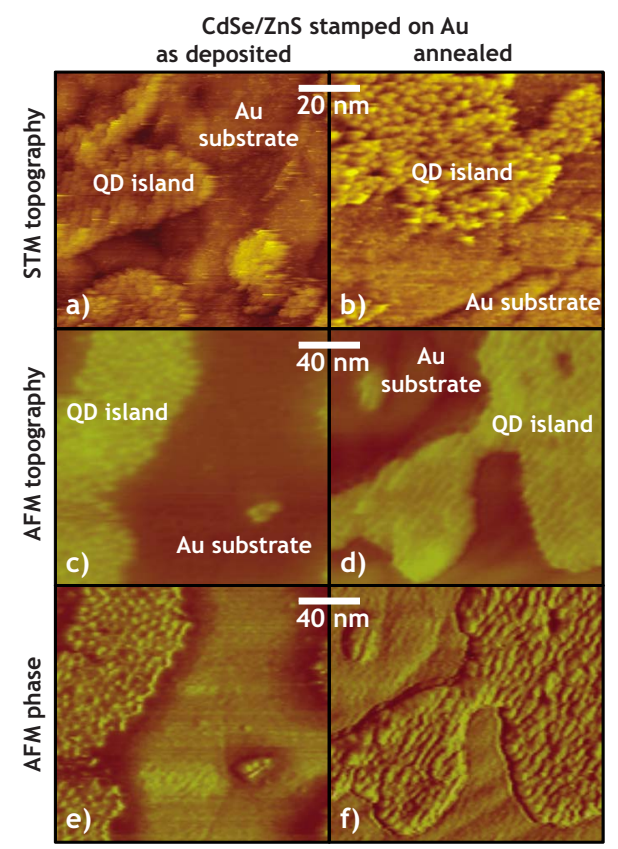

FIG. 2. (Color online) Scanning probe images of CdSe/ZnS QD on Au. Left column (a), (c), and (e) are as-deposited samples. Right column (b), (d), and (f) are images of samples annealed at $120{ }^{\circ} \mathrm{C}$ for $1 \mathrm{~h}$ in low vacuum (8 Torr). Top row (a) and (b) shows STM topography images, with a marked increase in clarity for the annealed samples. Middle row (c) and (d): AFM topography images show as-deposited and annealed average QD height of $6.6 \mathrm{~nm}$ and $6.1 \mathrm{~nm}$, respectively. Bottom row (e) and (f): AFM phase images show distinct contrast between the QD and Au sample areas.

tunnel through the conduction band; and (2) $R_{t i p-Q D}$ decreases, possibly allowing electrons to partially fill conduction states, resulting in a Coulomb staircase in $I V$ measurements. We do not observe this, and instead observe CB due to charges trapped in nontransport states of the QD. Unipolar transport obscures interpretation of the ZCR as a full voltage sweep does not probe both band edges. Our analysis is based on comparing only the band edge closest to the Fermi level (in our case, $E_{C, \mathrm{QD}}$ ) between subsequent spectra, and therefore, if unipolar transport occurs at negative biases, does not affect our analysis.

Ligand evaporation, by annealing, decreases the QDsubstrate distance, lowers $R_{\mathrm{QD}-\text { sub }}$ (increases $C_{\mathrm{QD}-\text { sub }}$ ), and increases the conductivity between the QD and the substrate. This is substantiated by the decrease in QD height measured in the AFM images and the much clearer STM topography image on annealed QDs [Fig. 2(b)]. We use a set-point tunneling current between 100 and $350 \mathrm{pA}$ when $V_{B}$ is in the range of 1.0-1.2 V, to maintain a tip-QD distance. All images and spectroscopy were collected in the absence of light.

\section{RESULTS AND DISCUSSION}

Tunneling spectra are taken in sequential pairs, first a decreasing bias sweep, immediately followed by an increasing bias sweep, as shown in Fig. 3. Before each set of spectra is collected, the feedback loop of the STM is closed for $1 \mathrm{~s}$ to

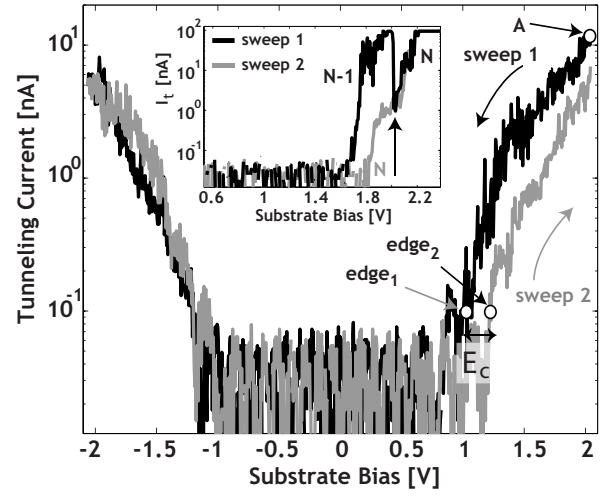

FIG. 3. Tunneling spectra are taken in sequential pairs, first a decreasing substrate bias $\left(V_{B}\right)$ sweep 1 , immediately followed by an increasing bias sweep 2. Point $\mathbf{A}$ is the detrap potential, the $V_{B}$ held for either 0 or $250 \mathrm{~ms}$, before making a measurement. Points edge and edge $e_{2}$ indicate the conduction-band edge of the QD and edge $_{2}$-edge $e_{1}$ is the charging energy, $E_{c}$. IV inset shows a single discharging event. $\mathrm{N}$ and $\mathrm{N}-1$ label the number of trapped charges on the QD.

stabilize the tip height above the QD. The feedback loop is then released and $V_{B}$ is set to the first data point (A in Fig. 3) and held there for either 0 or $250 \mathrm{~ms}$. Each spectra takes between 4 and $6 \mathrm{~s}$ to complete, depending on the sweep range. The sampling time per data point and the voltage step between data points is kept constant for all collected spectra.

We analyze each pair of spectra to determine the extent of the shift in the conduction-band edge by comparing points labeled edge $e_{1}$ and edge $e_{2}$ in Fig. 3. To determine the band edge, we fit an exponential curve to the $I V$ trace and then calculate the $V_{B}$ at which the tunneling current is approximately twice the background signal, ${ }^{36} \sim 100 \mathrm{pA}$. The shift in the band edge is due to $\mathrm{CB}$ and the potential-energy difference between the measured band edges $\left(\right.$ edge $_{2}$-edge $\left.{ }_{1}\right)$ is the charging energy, $E_{c}$.

The band-edge position of sweep 1, edge ${ }_{1}$, is always at a lower $V_{B}$ than edge ${ }_{2}$, implying that there is less Coulomb repulsion for sweep 1 . Therefore, we define the $V_{B}$ necessary to decrease the number of trapped charges on the $\mathrm{QD}$, before sweep 1 begins, as the detrap potential (DP); most often, this is the starting $V_{B}$ of sweep 1 (A in Fig. 3). In the event that the tunneling current exceeds the threshold of our preamplifier, $100 \mathrm{nA}$, causing a decrease in the bias between the tip and the substrate, we use the largest bias applied while the tunneling current is less than $100 \mathrm{nA}$.

\section{A. Charging-energy measurement}

In order to obtain a good statistical measurement of $E_{c}$ for a single charge-trapping/detrapping event in a close-packed monolayer of QDs, we analyzed thousands of pairs of sequential spectra. We specified a narrow range for the tip-QD distance, where the tip was positioned to ensure resonant tunneling, and we collected spectra from approximately 50 locations on two identically prepared samples. Figure 4 shows distributions of $E_{c}$ for three detrapping conditions: (a) $0 \mathrm{~s}$ wait time and high DP; (b) $250 \mathrm{~ms}$ wait time and low DP; 


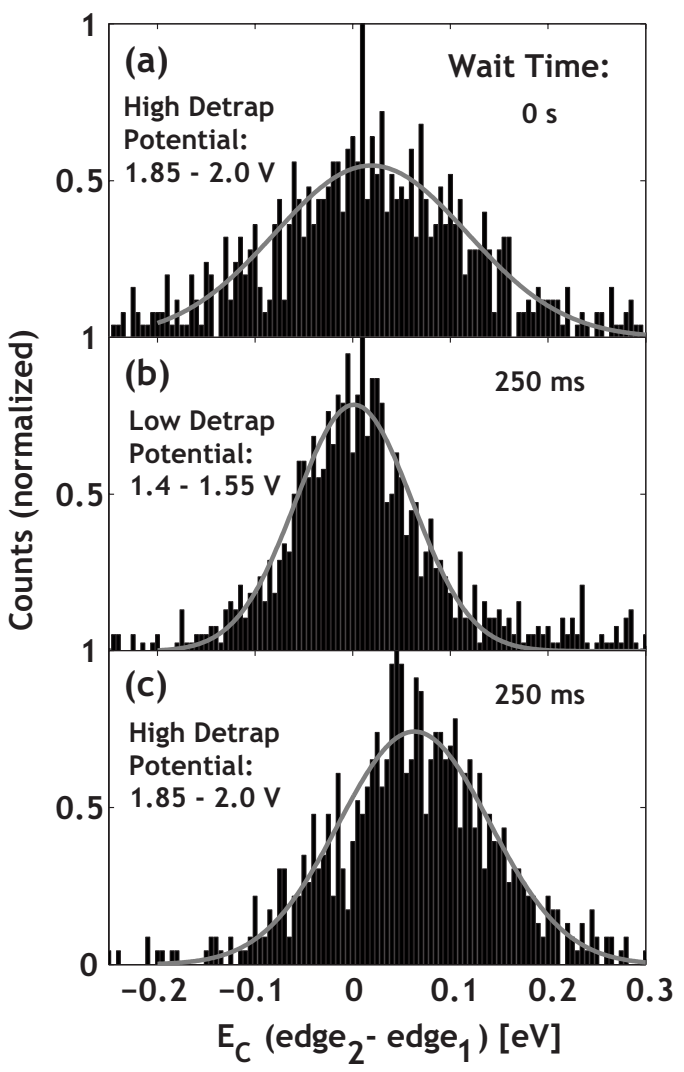

FIG. 4. Distributions of charging-energy measurements for three discharging conditions labeled in the figure. Histograms are composed of $k$ number of $E_{c}$ measurements and have a Gaussian peak, $\Gamma$, with $95 \%$ confidence bounds at (a) $k=722, \Gamma=(19 \pm 10) \mathrm{meV}$; (b) $k=1017, \Gamma=(1 \pm 4) \mathrm{meV}$; and (c) $k=692, \Gamma=(63 \pm 6) \mathrm{meV}$.

and (c) $250 \mathrm{~ms}$ wait time and high DP. Gaussians were fit to each distribution. The histogram of the high DP, $250 \mathrm{~ms}$ wait time $E_{c}$ data [Fig. 4(c)] shows a notably larger peak value of $E_{c},(63 \pm 6) \mathrm{meV}$, than the $0 \mathrm{~s}$ wait time, high DP, $(19 \pm 10) \mathrm{meV}$, or $250 \mathrm{~ms}$ wait time, low DP distributions, $(1 \pm 4) \mathrm{meV}$. It appears that the QD changes charge state when detrap potential of $1.85 \mathrm{~V}$ is applied for $250 \mathrm{~ms}$, however if either the detrap potential is lower or the wait time is lower then there is no change in the charge state.

The distributions in Fig. 4 have an average FWHM of $(186 \pm 47) \mathrm{meV}$, which we attribute to variations in the tip-QD distance due to tip drift while the feedback loop is off. Histograms of the negative band-edge potential, which does not demonstrate any charging effect, have nearly identical FWHM, (183 \pm 52$) \mathrm{meV}$, as the positive band-edge potential shown in Fig. 4. Increased broadening is consistent with an increased amount of time since the feedback loop was turned off. In Fig. 6(b), the distribution of edge measurements for sweep 1 are much narrower than sweep 2 since the feedback loop is engaged only prior to sweep 1 . In addition, the charging energy of a QD is affected by the number and charge state of neighboring QDs, which changes the capacitive coupling to the environment.

To understand how the magnitude of the DP affects the charged state of the QD, and thus $E_{c}$, in Fig. 5 we plot the $E_{c}$ for a range of DP values, for both the 0 and $250 \mathrm{~ms}$ wait

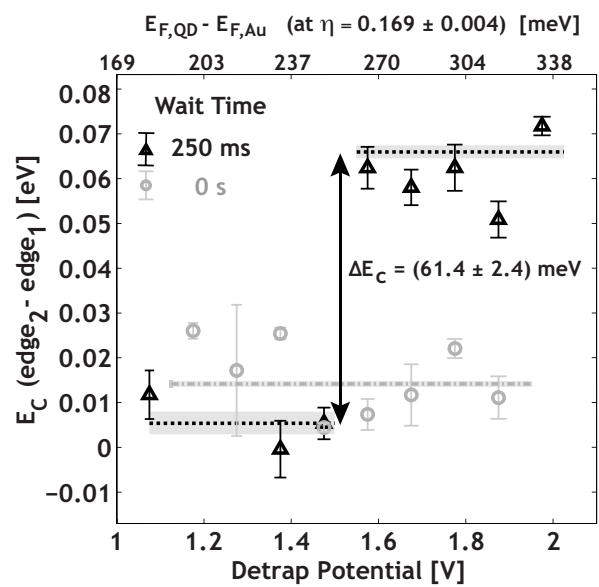

FIG. 5. $E_{c}$ as a function of substrate bias (detrap potential, bottom axis) and bias drop between the QD and the Au substrate $\left(E_{F, \mathrm{QD}}-E_{F, \mathrm{Au}}\right.$, top axis) for a wait time of $0 \mathrm{~s}$ (gray circles) and 250 ms (black triangles). $\Delta E_{c}$ is the difference between the weighted averages of the $250 \mathrm{~ms}$ data, above and below DP $\sim 1.55 \mathrm{~V}$ $\left(E_{F, \mathrm{QD}}-E_{F, \mathrm{Au}} \sim 262 \mathrm{meV}\right)$.

time. Each data point is the average $E_{c}$ value for a narrow range of detrap potentials, where the error bars are the standard error. The weighted average and standard deviation of the $250 \mathrm{~ms}$ data shows a shift from $(5.5 \pm 1.3)$ to $(66.9 \pm 1.1) \mathrm{meV}$ at a DP $\sim 1.55 \mathrm{~V}$. The $0 \mathrm{~s}$ data shows an average $E_{c}$ measurement of $(14.8 \pm 1.1) \mathrm{meV}$ over all DPs. The $E_{c}$ for a typical QD in our study is the difference between the low and high DP distributions, $(61.4 \pm 2.4) \mathrm{meV}$. The critical DP at a wait time of $250 \mathrm{~ms}$ is $\sim 1.55 \mathrm{~V}$. We observe charge detrapping in $\sim 50 \%$ of the measurements at $\mathrm{DP}=1.4 \mathrm{~V}$, when the wait time is extended to $4 \mathrm{~s}$.

\section{B. Charge state}

The single charging event depicted in the inset of Fig. 3 is an example of a QD changing its charge state in the middle of the $I V$ sweep. In the sequence shown, a forward sweep (sweep 1) starts from a high bias in an $\mathrm{N}$ state, indicating that there are $\mathrm{N}$ excess electrons on the QD. The QD loses an electron at a bias indicated by the arrow $(2.05 \mathrm{~V})$, changing its charge state to $\mathrm{N}-1$. In the zero-conductance region, it appears that the QD retraps an electron. As sweep 2 passes through the band edge, it is in the $\mathrm{N}$ state. This spontaneous change in the charge state, from $\mathrm{N}$ to $\mathrm{N}-1$, is observed in numerous consecutive sweeps and it suggests that we are observing single electron detrapping events. The charged state time for this particular QD is estimated to be $0.5 \mathrm{~s}$, which corresponds to the time that sweep 1 remained in the $\mathrm{N}$ state. Such a long charge retention time is indicative of a large potential barrier for initiating detrapping events.

In any given current-voltage sweep, the QD may or may not change its charge state, necessitating the sequential attainment of a spectra with a known charge state, in order to verify a Coulomb-blockade event. Plotting the distribution of edge $_{1}$ and edge ${ }_{2}$ values, above and below the critical detrap potential of $V_{B}=1.55 \mathrm{~V}$, demonstrates the charged state of the QD. In Fig. 6, we show the average conduction-band 


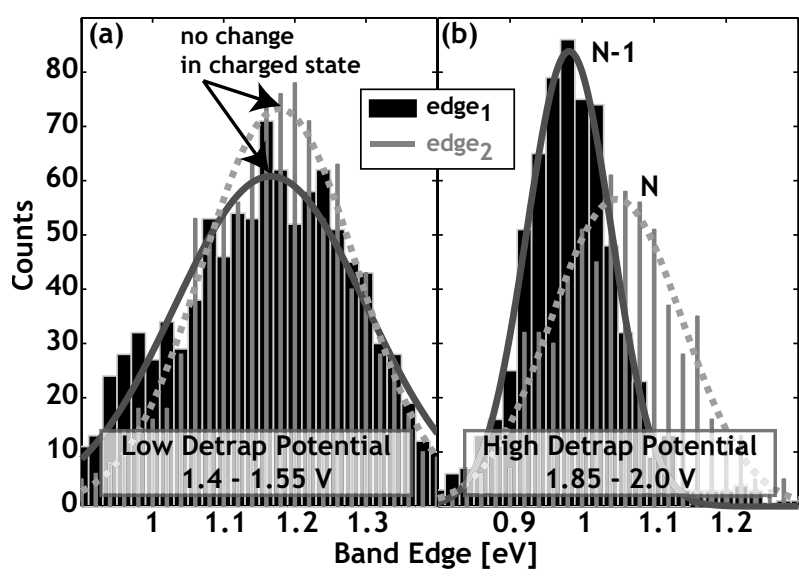

FIG. 6. Distributions of conduction-band edge energy, labeled

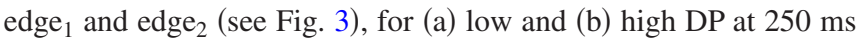
wait time. At high DPs, the distributions of edge ${ }_{1}$ and edge $e_{2}$ are separated by $(66 \pm 13) \mathrm{meV}$, in contrast to the low DP separation of $(12 \pm 16) \mathrm{meV}$. The labels $\mathrm{N}$ and $\mathrm{N}-1$ are indicative of the number of excess charges on the QD.

edge energy, for a wait time of $250 \mathrm{~ms}$, at (a) low and (b) high DPs. The $0 \mathrm{~s}$ wait time data showed no change in the band-edge position for high and low detrap potential, indicating that the charge state of the QD remained constant. The $250 \mathrm{~ms}$ wait time data shows remarkably different behavior for high and low DPs: at high DPs [Fig. 6(b)], edge $_{1}$ (wide, black bars) is $(66 \pm 13) \mathrm{meV}$ lower in energy than edge ${ }_{2}$ (narrow, gray bars) while at low DPs [Fig. 6(a)], the distributions of edge ${ }_{1}$ and edge $e_{2}$ essentially overlap, with negligible energy separation of $(12 \pm 16) \mathrm{meV}$. Average tip-QD distance is greater for the low DP measurements $\left[I_{t}=(131 \pm 47) \mathrm{pA}, V_{B}=(1.125 \pm 0.009) \mathrm{V}\right]$ than the high DP measurements $\left[I_{t}=(228 \pm 16) \mathrm{pA}, V_{B}=(1.113 \pm 0.020) \mathrm{V}\right]$, thus increasing the absolute band-edge energy. The high DP data is labeled as $\mathrm{N}-1$ and $\mathrm{N}$ to indicate that the QD is charged with $\mathrm{N}$ electrons at zero bias and one electron is removed from the QD after applying a high $V_{B}(>1.55 \mathrm{~V})$ for $250 \mathrm{~ms}$.

\section{Charging-energy calculation}

Many previous publications have discussed methods of experimentally measuring $E_{c}$ of nanocrystals using a scanning tunneling microscope, ${ }^{24,37-39}$ as well as calculating $E_{c}{ }^{34,35,40}$ The charge-trapping center in CdSe/ZnS QDs likely exists at either the core/shell interface, due to a dangling bond on $\mathrm{Se}^{22,28}$ or on the shell surface, due to incomplete ligand passivation of $\mathrm{ZnS}$ surface atoms. The exact location of the trapped charge does not appreciably affect the charging energy of the QD, however it does effect the trapping/detrapping probability. Annealing the QD samples may increase the number of nonpassivated surface states.

To estimate $E_{c}$, we employ the equation $E_{c}=e^{2} / C{ }^{41}$ where $e$ is the charge of an electron and $C$ is sum of the capacitances between the QD core and its surrounding: $C_{t i p-\mathrm{QD}}+C_{\mathrm{QD}-s u b}+n C_{\mathrm{QD}-\mathrm{QD}} \cdot n$ is the number of nearestneighbor QDs. Figure 7(a) is a pictorial representation of the

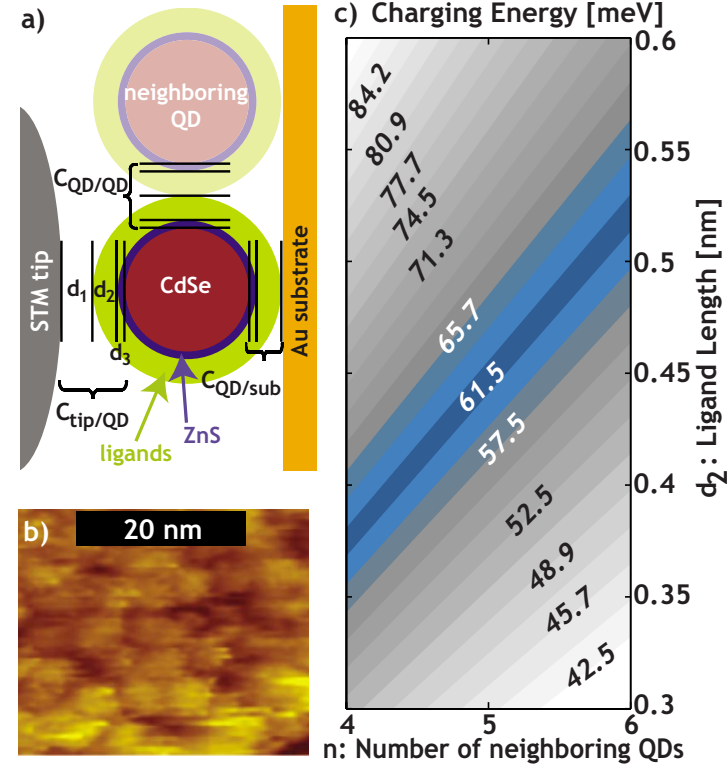

FIG. 7. (Color online) Charging-energy $\left(E_{c}\right)$ calculation. (a) Schematic of capacitance between the QD and substrate, tip, and neighboring QDs. (b) An example of a probed QD and five to six nearest neighbors in a hexagonal close pack structure (STM image). (c) $E_{c}$ contour plot for a trapped charge at the core/shell interface, plotted versus ligand length and number of neighboring quantum dots. Measured $E_{c},(61.4 \pm 2.4) \mathrm{meV}$, is highlighted on the contour plot.

relevant capacitors between the QD core, a plausible location for the trapped charge, and its surroundings. The capacitances between the core and the tip, substrate, and nearestneighbor QDs are modeled as two or three parallel-plate capacitors in series. We estimate the $\mathrm{ZnS}$ shell, $d_{3}$, to be a monolayer, corresponding to an average thickness of 0.31 $\mathrm{nm}$. The length of the ligands, $d_{2}$, is estimated to be $0.3-0.6$ $\mathrm{nm}$ and we plot the calculated $E_{c}$ for a range of $d_{2}$ values in Fig. $7(\mathrm{c})$. We estimate the vacuum gap, $d_{1}$, to be around 1 $\mathrm{nm},{ }^{42}$ and found that values ranging from 0.8 to $1.2 \mathrm{~nm}$ had very little effect on the calculation of $E_{c}$ since the dominant capacitive coupling is between QDs. We used the dielectric constant values of $\epsilon / \epsilon_{0}=2.1,9$, and 8 for the ligands, $\mathrm{ZnS}$, and CdSe, respectively. Our STM [see Fig. 7(b)] and AFM [see Fig. 2(e)] images show close packing of the QD monolayer islands, with most QDs surrounded by five nearest neighbors.

Figure 7(c) is contour plot of the $E_{c}$, for adding one electron to the QD at the interface between the core and the shell, as a function of the ligand thickness and the number of neighboring QDs. We do not explicitly take into account the localized nature of the trapped charge, however we believe that the trap states accessible in our experiment are only those closest to the Au substrate. Since we measured an $E_{c}$ of $(61.4 \pm 2.4) \mathrm{meV}$ [see Figs. 5 and 6], the shaded region of Fig. 7(c) shows that this corresponds to a ligand length of $(0.45 \pm 0.02) \mathrm{nm}$ for an average of five nearest-neighbor QDs. For $d_{1}=1 \mathrm{~nm}$ and $d_{2}=0.45 \mathrm{~nm}, C_{\mathrm{QD}-\mathrm{sub}}$ and $C_{t i p-\mathrm{QD}}$ are $(0.70 \pm 0.03) \mathrm{aF}$ and $(0.143 \pm 0.001) \mathrm{aF}$, respectively. We then find $\eta$, the portion of $V_{B}$ that drops between the QD and substrate, to be $0.169 \pm 0.004$. If the trapped charge is 


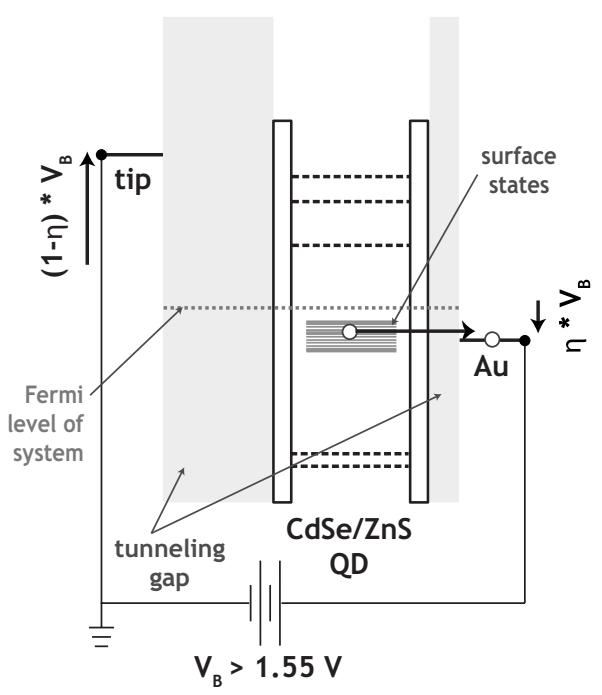

FIG. 8. Tunneling diagram for detrapping a charge from the QD at $V_{B}>1.55 \mathrm{~V}$. At $V_{B}=0 \mathrm{~V}$, the Fermi level of Au and the W tip are in equilibrium and lie slightly above the surface-state band, trapping charge(s) on the QD. The distribution of $V_{B}$ over the two tunnel gaps $\left(\eta * V_{B}\right.$ over the QD-Au gap) causes the Fermi level of the $\mathrm{Au}$ to drop slightly from its equilibrium energy, thus exposing empty states on the Au surface to the trapped charge.

located at the outer surface of the shell, $d_{2}$ and $\eta$ are $(0.66 \pm 0.03) \mathrm{nm}$ and $0.197 \pm .005$. Transmission electron micrographs of ligand-coated nanocrystals demonstrate that the physical length of ligands outside of solution is on the order of $1 \mathrm{~nm},{ }^{22,43}$ ligand length is expected to decrease upon annealing.

The charging energy for an isolated QD $(n=0)$ on a Au thin film, with the same tunneling parameters as used above, is $200 \mathrm{meV}$. Attempts made to obtain $I V$ spectra on isolated QDs were precluded by the significant lateral drift over the course of the experiment: hundreds of spectra and tens of minutes.

\section{Charge-trapping mechanism}

We have established that near zero bias, a charge is trapped on the QD and extended exposure $(250 \mathrm{~ms})$ to high positive $V_{B}(>1.55 \mathrm{~V})$ has a high likelihood of removing an electron from the QD (Fig. 5). Figure 8 demonstrates how trap states in the band gap of the QD could be occupied near $V_{B}=0 \mathrm{~V}$ (Fermi level of the Au-film substrate is above the trap states) and have a high tunneling probability at $V_{B}>1.55 \mathrm{~V}$ (Fermi level of the Au-film substrate is resonant with the trap states). The band diagram of the core/shell QD in relation to the $\mathrm{W}$ tip and Au-film substrate shown in Fig. 8 was derived from the following measurements. The $\mathrm{CdSe}$ valence-band edge was determined by ultraviolet photoelectron spectroscopy (UPS), ${ }^{44}$ and found to be $-6.8 \mathrm{eV}$. The $\mathrm{CdSe}$ conduction-band edge was determined by the photonic band gap, $2 \mathrm{eV}(-4.8 \mathrm{eV})$. The valence- and conductionband edges of the $\mathrm{ZnS}$ shell, $-7.4 \mathrm{eV}$ and $-3.4 \mathrm{eV}$, were determined from thin-film UPS and photoluminescence measurements, respectively. The Fermi level of $\mathrm{Au}$ and $\mathrm{W}$ are $-5.1 \mathrm{eV}$ and $-4.5 \mathrm{eV}$, respectively.
There are two likely locations for the trapped charge to reside on the QD: at the core/shell interface or on the shell surface. Past luminescence ${ }^{45-48}$ and conductivity studies $^{32,49,50}$ of quantum dots have found that surface states in the band gap play a significant role is quenching photon emission and enhancing charge transport. Though passivating the core surface with organic ligands or an inorganic shell reduces the number of dangling bonds, tight-binding calculations show that even after surface reconstruction, one of the surface Se dangling bonds has a significant density of states in the band gap. ${ }^{51}$ A number of experimental studies place the CdSe core surface-state band ${ }^{52} 400-700 \mathrm{meV}$ below the conduction-band edge ${ }^{32,50,53}$ including time-resolved fluorescence studies that show broad, redshifted emission. ${ }^{48,54}$

We estimate the Fermi level of the tip-QD-substrate system from the measurement of the conduction-band edge, edge $_{1}$, in Fig. 3. The average measured potential of the edge conduction-band edge is $(0.974 \pm 0.001) \mathrm{V}$. Applying $1-\eta$ $=0.831$ (see Sec. III C), $E_{c}-E_{F}=(0.809 \pm 0.001) \mathrm{eV}$. The tunnel barrier between the QD and the Au film is much thinner than between the STM tip and QD, making the former the dominant charge-transfer barrier. During sweep 1, the conduction-band edge $\left(\right.$ edge $\left._{1}\right)$ is measured (near $V_{B}=1 \mathrm{~V}$ ) while the QD is still in $\mathrm{N}-1$ charge state. Near $V_{B}=0 \mathrm{~V}$, electrons can easily tunnel from the Au film to the QD surface states but cannot tunnel out of the QD to either the substrate or tip, and thus when $V_{B}$ is increased again (sweep 2 ), the conductivity is lower due to Coulomb repulsion from the trapped electron, and the band-edge energy $\left(\right.$ edge $\left._{2}\right)$ is measured at a higher $V_{B}$ than for edge $e_{1}$.

At large positive biases $\left(V_{B}>1.55 \mathrm{~V}\right)$, the asymmetric voltage drop across the two tunnel barriers causes a slight decrease in the potential of $E_{F, A u}$ relative to the QD, thus aligning the empty states in the Au with the surface states in the QD and allowing the trapped charge to tunnel off of the QD (charge state: N-1). The top axis of Fig. 5 applies our estimated $\eta$ to $V_{B}$ and shows that the onset of detrapping occurs at $E_{F, \mathrm{QD}}-E_{F, \mathrm{Au}}=260 \mathrm{meV}, 1.06 \mathrm{eV}$ below the conduction-band edge of CdSe. The wait time of $250 \mathrm{~ms}$ is necessary because the trapped electron-tunneling probability is very low.

Previous studies have observed long-lived ( $>1$ s) charge occupation of trap states in $\mathrm{CdSe} / \mathrm{ZnS}$ QDs during blinking experiments. ${ }^{55,56}$ These are manifested as "off" time in the fluorescence detection of continually excited QDs, and have been observed for times as long as $100 \mathrm{~s}$.

\section{SUMMARY}

We demonstrate a method for determining the charging energy and charge state of nanocrystal QDs and applied it to $\mathrm{CdSe} / \mathrm{ZnS}$ core/shell QDs. We show that a long-lived trap state on $\mathrm{CdSe} / \mathrm{ZnS}$ can be probed with a scanning tunneling microscope at room temperature and the charging energy for individual QDs in a close-packed monolayer, $(61.4 \pm 2.4) \mathrm{meV}$, can be determined by observing the $\mathrm{CB}$ of subsequent spectra. Our calculation of the energy necessary to add one charge to the quantum dot corresponds 
closely to the assumed ligand thickness and number of neighboring QDs.

We also identify the necessary potential to change the charge state of the quantum dot, $1.55 \mathrm{~V}$, with a wait time of $250 \mathrm{~ms}$. Our analysis of the double-barrier tunnel junction DBTJ yields an approximate location for the trap states in the band gap of the QD. We find that $E_{F, \text { Au }}$ moves by $-\eta V_{B}$, and at $V_{B}=1.55 \mathrm{~V}, E_{F, \mathrm{Au}}$ is $1.06 \mathrm{eV}$ below the conductionband edge of CdSe. This is supported by a depiction of the energy-band alignment of the QD and Au film in Fig. 8, which demonstrates that at $V_{B}>1.55 \mathrm{~V}$, the surface-state band is above the Fermi level of the Au film. It is possible that our electrical detection of a trapped charge is analogous to observing the off state in blinking experiments. Further studies could help quantify the trapped charge lifetime under various electric field conditions.

\section{ACKNOWLEDGMENTS}

We thank QD Vision, Inc. for supplying the QDs used in this work. Samples were prepared by use of MRSEC Shared Experimental Facilities at MIT, supported by the National Science Foundation (NSF) (Grant No. DMR-02-13282) and the Institute for Soldier Nanotechnologies (Contract No. DAAD-19-02-0002). Microscope measurements were supported by the NSF (Contract No. NSF/PHY 06-46094) through the Nanoscale Science and Engineering Center and through the use of facilities at the Rowland Institute at Harvard. Support from NASA-Ames Research Center (NNA04CK42A) funded the microscope fabrication. V. Bulović acknowledges financial support from DOE funding through the Solar America Program Grant No. DE-FG3608GO18007.

*marissa.hummon@ post.harvard.edu

${ }^{1}$ J. M. Caruge, J. E. Halpert, V. Bulović, and M. G. Bawendi, Nano Lett. 6, 2991 (2006).

${ }^{2}$ S. Chaudhary, M. Ozkan, and W. C. W. Chan, Appl. Phys. Lett. 84, 2925 (2004).

${ }^{3}$ S. Coe, W. K. Woo, M. Bawendi, and V. Bulović, Nature (London) 420, 800 (2002).

${ }^{4}$ S. Coe-Sullivan, W. K. Woo, J. S. Steckel, M. Bawendi, and V. Bulović, Org. Electron. 4, 123 (2003).

${ }^{5}$ D. I. Son, J. H. Kim, D. H. Park, W. K. Choi, F. Li, J. H. Ham, and T. W. Kim, Nanotechnology 19, 055204 (2008).

${ }^{6}$ M. V. Jarosz, V. J. Porter, B. R. Fisher, M. A. Kastner, and M. G. Bawendi, Phys. Rev. B 70, 195327 (2004).

${ }^{7}$ J. M. Caruge, J. E. Halpert, V. Wood, V. Bulović, and M. G. Bawendi, Nat. Photonics 2, 247 (2008).

${ }^{8}$ P. O. Anikeeva, C. F. Madigan, J. E. Halpert, M. G. Bawendi, and V. Bulović, Phys. Rev. B 78, 085434 (2008).

${ }^{9}$ P. P. Jha and P. Guyot-Sionnest, J. Phys. Chem. C 111, 15440 (2007).

${ }^{10}$ T. S. Mentzel, V. J. Porter, S. Geyer, K. MacLean, M. G. Bawendi, and M. A. Kastner, Phys. Rev. B 77, 075316 (2008).

${ }^{11}$ H. Huang, A. Dorn, V. Bulović, and M. G. Bawendi, Appl. Phys. Lett. 90, 023110 (2007).

${ }^{12}$ H. Huang, A. Dorn, G. P. Nair, V. Bulović, and M. G. Bawendi, Nano Lett. 7, 3781 (2007).

${ }^{13}$ O. Millo, D. Katz, Y. W. Cao, and U. Banin, Phys. Rev. B 61, 16773 (2000).

${ }^{14}$ E. P. A. M. Bakkers and D. Vanmaekelbergh, Phys. Rev. B 62, R7743 (2000).

${ }^{15}$ O. Millo, D. Katz, Y. W. Cao, and U. Banin, Phys. Rev. Lett. 86, 5751 (2001)

${ }^{16}$ E. P. A. M. Bakkers, Z. Hens, A. Zunger, A. Franceschetti, L. Kouwenhoven, L. Gurevich, and D. Vanmaekelbergh, Nano Lett. 1, 551 (2001).

${ }^{17}$ M. Soreni-Harari, N. Yaacobi-Gross, D. Steiner, A. Aharoni, U. Banin, O. Millo, and N. Tessler, Nano Lett. 8, 678 (2008).

${ }^{18}$ L. Jdira, P. Liljeroth, E. Stoffels, D. Vanmaekelbergh, and S. Speller, Phys. Rev. B 73, 115305 (2006).

${ }^{19}$ K. Walzer, E. Marx, N. Greenham, and K. Stokbro, Surf. Sci.

532-535, 795 (2003).

${ }^{20}$ R. Bernard, G. Comtet, G. Dujardin, V. Huc, and A. Mayne, Appl. Phys. Lett. 87, 053114 (2005).

${ }^{21}$ D. Steiner, T. Mokari, U. Banin, and O. Millo, Phys. Rev. Lett. 95, 056805 (2005).

${ }^{22}$ M. Shim and P. Guyot-Sionnest, J. Chem. Phys. 111, 6955 (1999).

${ }^{23}$ U. Banin, Y. W. Cao, D. Katz, and O. Millo, Nature (London) 400, 542 (1999).

${ }^{24}$ B. Alperson, I. Rubinstein, G. Hodes, D. Porath, and O. Millo, Appl. Phys. Lett. 75, 1751 (1999).

${ }^{25}$ D. V. Averin and K. K. Likharev, J. Low Temp. Phys. 62, 345 (1986).

${ }^{26}$ R. Wilkins, E. Ben-Jacob, and R. C. Jaklevic, Phys. Rev. Lett. 63, 801 (1989).

${ }^{27}$ S. Tiwari, F. Rana, H. Hanafi, A. Hartstein, E. F. Crabbe, and K. Chan, Appl. Phys. Lett. 68, 1377 (1996).

${ }^{28}$ B. O. Dabbousi, J. Rodriguez Viejo, F. V. Mikulec, J. R. Heine, H. Mattoussi, R. Ober, K. F. Jensen, and M. G. Bawendi, J. Phys. Chem. B 101, 9463 (1997).

${ }^{29}$ L. Kim, P. O. Anikeeva, S. A. Coe-Sullivan, J. S. Steckel, M. G. Bawendi, and V. Bulović, Nano Lett. 8, 4513 (2008).

${ }^{30}$ J. J. Blackstock, Z. Y. Li, M. R. Freeman, and D. R. Stewart, Surf. Sci. 546, 87 (2003).

${ }^{31}$ V. J. Porter, T. S. Mentzel, S. Charpentier, M. A. Kastner, and M. G. Bawendi, Phys. Rev. B 73, 155303 (2006).

${ }^{32}$ V. J. Porter, S. Geyer, J. E. Halpert, M. A. Kastner, and M. G. Bawendi, J. Phys. Chem. C 112, 2308 (2008).

${ }^{33}$ P. Liljeroth, L. Jdira, K. Overgaag, B. Grandidier, S. Speller, and D. Vanmaekelbergh, Phys. Chem. Chem. Phys. 8, 3845 (2006).

${ }^{34}$ Y. M. Niquet, C. Delerue, G. Allan, and M. Lannoo, Phys. Rev. B 65, 165334 (2002).

${ }^{35}$ U. Banin and O. Millo, Annu. Rev. Phys. Chem. 54, 465 (2003).

${ }^{36} \sigma$ is the standard deviation of the background signal. The lock-in amplifier used to record the differential conductance has a background signal of $50 \mathrm{pA}$.

${ }^{37}$ B. Zaknoon, G. Bahir, C. Saguy, R. Edrei, A. Hoffman, R. A. Rao, R. Muralidhar, and K. Chang, Nano Lett. 8, 1689 (2008).

${ }^{38}$ B. Li, C. G. Zeng, J. Zhao, J. L. Yang, J. G. Hou, and Q. S. Zhu, 
J. Chem. Phys. 124, 064709 (2006).

${ }^{39}$ O. Millo, D. Katz, Y. Levi, Y. W. Cao, and U. Banin, J. Low Temp. Phys. 118, 365 (2000).

${ }^{40}$ D. V. Averin, A. N. Korotkov, and K. K. Likharev, Phys. Rev. B 44, 6199 (1991).

${ }^{41}$ L. P. Kouwenhoven, C. M. Marcus, P. L. McEuen, S. Tarucha, R. M. Westervelt, and N. S. Wingreen, in Mesoscopic Electron Transport, Series E: Applied Sciences, edited by L. L. Sohn, L. P. Kouwenhoven, and G. Schön (Kluwer Academic, Dordrecht, 1997), Vol. 345, pp. 105-214.

${ }^{42}$ Tunneling gap was estimated by solving $I \sim(1$ $-\eta) V_{B} \exp ^{-1.025} \bar{\top} \Phi d_{t i p / \mathrm{QD}}$ for the setpoint current and $V_{B}$. The calculated value of $d_{t i p / \mathrm{QD}}$ is both a function of the voltage division and a factor in determining the voltage division, thus it is solved for iteratively.

${ }^{43}$ U. Banin, C. J. Lee, A. A. Guzelian, A. V. Kadavanich, A. P. Alivisatos, W. Jaskolski, G. W. Bryant, A. L. Efros, and M. Rosen, J. Chem. Phys. 109, 2306 (1998).

${ }^{44}$ UPS measurements were performed on thin-film $\mathrm{ZnS}$ and similarly prepared $\mathrm{CdSe} / \mathrm{ZnS} \mathrm{QD}$ on Au substrate samples. Measured ionization energies of $(7.4 \pm 0.2) \mathrm{eV}$ and $(6.9 \pm 0.2) \mathrm{eV}$ for the $\mathrm{ZnS}$ and $\mathrm{CdSe} / \mathrm{ZnS}$ QD films were obtained, respectively. The $6.9 \mathrm{eV}$ measurement for the CdSe/ZnS QDs likely represents an average of the $\mathrm{ZnS}$ shell and CdSe core contributions to the valence states. We, therefore, place the valence-band edge of the core at $6.8 \mathrm{eV}$.

${ }^{45}$ M. Jones, J. Nedeljkovic, R. J. Ellingson, A. J. Nozik, and G. Rumbles, J. Phys. Chem. B 107, 11346 (2003).

${ }^{46}$ B. Nikoobakht, C. Burda, M. Braun, M. Hun, and M. A. ElSayed, Photochem. Photobiol. 75, 591 (2002).

${ }^{47}$ M. Kuno, J. K. Lee, B. O. Dabbousi, F. V. Mikulec, and M. G. Bawendi, J. Chem. Phys. 106, 9869 (1997).

${ }^{48}$ M. G. Bawendi, P. J. Carroll, W. L. Wilson, and L. E. Brus, J. Chem. Phys. 96, 946 (1992).

${ }^{49}$ R. A. M. Hikmet, D. V. Talapin, and H. Weller, J. Appl. Phys. 93, 3509 (2003).

${ }^{50}$ B. Alperson, I. Rubinstein, and G. Hodes, Phys. Rev. B 63, 081303(R) (2001).

${ }^{51}$ S. Pokrant and K. B. Whaley, Eur. Phys. J. D 6, 255 (1999).

${ }^{52}$ P. A. Frantsuzov and R. A. Marcus, Phys. Rev. B 72, 155321 (2005).

${ }^{53}$ N. Myung, Y. Bae, and A. J. Bard, Nano Lett. 3, 1053 (2003).

${ }^{54}$ D. F. Underwood, T. Kippeny, and S. J. Rosenthal, J. Phys. Chem. B 105, 436 (2001).

${ }^{55}$ M. Kuno, D. P. Fromm, H. F. Hamann, A. Gallagher, and D. J. Nesbitt, J. Chem. Phys. 112, 3117 (2000).

${ }^{56}$ M. Kuno, D. P. Fromm, H. F. Hamann, A. Gallagher, and D. J. Nesbitt, J. Chem. Phys. 115, 1028 (2001). 